\title{
Compensation Method of Position Signal Error with Misaligned Hall-Effect Sensors of BLDC Motor
}

\author{
Joon Sung Park*, Jun-Hyuk Choi** and Ju Lee ${ }^{\dagger}$
}

\begin{abstract}
This paper presents an improved approach for compensating rotor position signal displacement in brushless DC (BLDC) motors with misaligned hall-effect sensors. Typically, the halleffect sensors in BLDC motors are located in each phase and positioned exactly 120 electrical degrees apart. However, limitations in mechanical tolerances make it difficult to place hall-effect sensors at the correct location. In this paper, a position error compensator to counteract the hall-effect sensor positioning error is proposed. The proposed position error compensator uses least squares error analysis to adjust the relative position error and back-EMF information to reduce the absolute offset error. The effectiveness of the proposed approach is verified through several experiments.
\end{abstract}

Keywords: Brushless dc (BLDC) motor, Hall-effect sensor, Position estimation, Least square error, Back-EMF

\section{Introduction}

Brushless DC (BLDC) motors have numerous advantages, including lower maintenance costs, simple drive techniques, wide speed ranges, high power per volume, and fewer environment-related restrictions. Because of these advantages, BLDC motors are widely used in many industries, including robotics, home appliances, vehicles, office automation, and industrial automation systems, and future demand for BLDC motors is expected to rise continuously. Hall-effect sensors are usually used in BLDC motors. Such sensors, however, may be installed in incorrect positions owing to mechanical, mounting, or production problems. When hall-effect sensors are misplaced, they can cause current and torque ripple, noise, and incorrect position control. In particular, such problems tend to occur in BLDC motors with high numbers of poles and small sizes.

The theory of BLDC control has been investigated thoroughly in the literature [1-3]. Most of the studies of the BLDC motor assume that correct positioning of the hall sensors means that they are spaced at intervals of exactly 120 electrical degrees, which may not be true for many of the low-cost, low-precision motors that are mass produced on the market [4].

The effects of misaligned hall sensors on stator currents and resulting torque are demonstrated in $[4,5]$. In order to reduce such effects, various approaches have been developed [4-9], with the methods developed in [4-7] concentrating

$\dagger$ Corresponding Author: Dept. of Electrical Engineering, Hanyang University, Korea. (julee@hanyang.ac.kr)

* Dept. of Electrical Engineering, Hanyang University, Korea. (parkjs@keti.re.kr)

** Intelligent Mechatronics Research Center, Korea Electronics Technology Institute, Korea. (cjh@keti.re.kr)

Received: November 6, 2015; Accepted: December 22, 2015 on the effects of using different combinations of hall sensors. The use of typical approaches for estimating rotor position from filtered hall-effect sensor signals, such as averaging and extrapolation, can ensure that the hall-effect sensors are electrically spaced at 120 degree intervals; however, the absolute positions of the hall-effect sensors cannot be aligned for each phase. Some recent approaches for compensating the misalignment effect were introduced in $[8,9]$. [8] demonstrated a vector-tracking observer that uses a quantized rotating position vector at $60^{\circ}$ obtained from hall sensor signals. In [9], a vector-tracking observer that uses a feedforward input of the average rotor speed to derive the rotor position with high-resolution was presented. However, both methods require the use of correct phase current and motor constants as they are based on a mechanical model of the motor; correspondingly, their

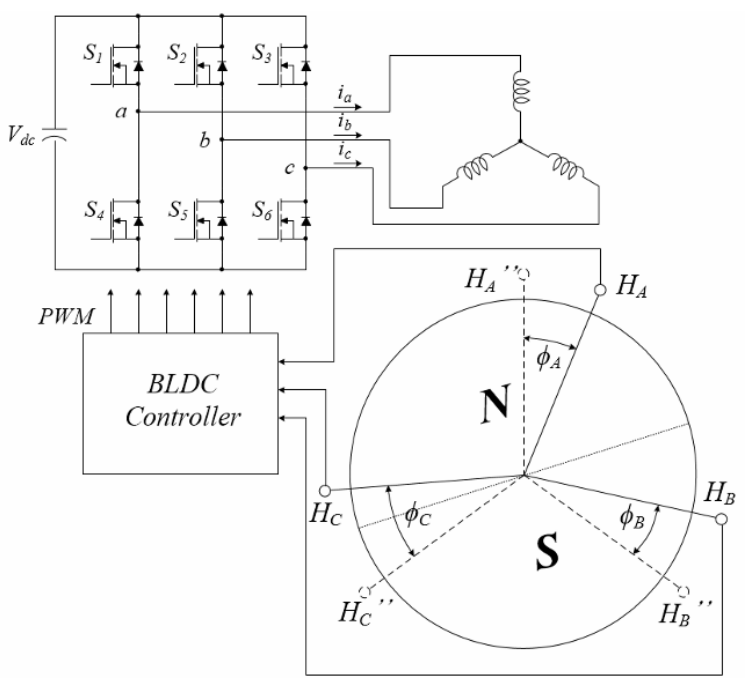

Fig. 1. BLDC drive configuration with misaligned halleffect sensors 
position estimation accuracy may be easily affected by load variations, phase current, and motor constants, which make the system more complex and render these methods unsuitable for use under simple control, e.g., BLDC square-wave control.

In this paper, a position signal displacement error compensation method for misaligned hall-effect sensors is proposed. In the proposed method, the output of the balancing unit, which is based on the least squares method $[10,11]$ and measured using the combination of hall signals, is compared with the zero crossing point (ZCP) detected by the offset unit. This elegant combination of activity by the balancing and offset units enhances the accuracy of angle estimation.

\section{Rotor Position Estimation}

As illustrated in Fig. 2, BLDC drives using hall-effect sensors are operated by output signals. When the hall-effect sensors are ideally placed 120 electrical degrees apart, the waveforms of their output signals can be divided into six 60-degree electrical sectors. However, if the hall-effect sensors are misaligned, the combination of actual sensor output will be irregularly repeated.

The quantities $\phi_{A}, \phi_{B}$, and $\phi_{C}$, which denote the respective misplacement angles, can be derived as follows:

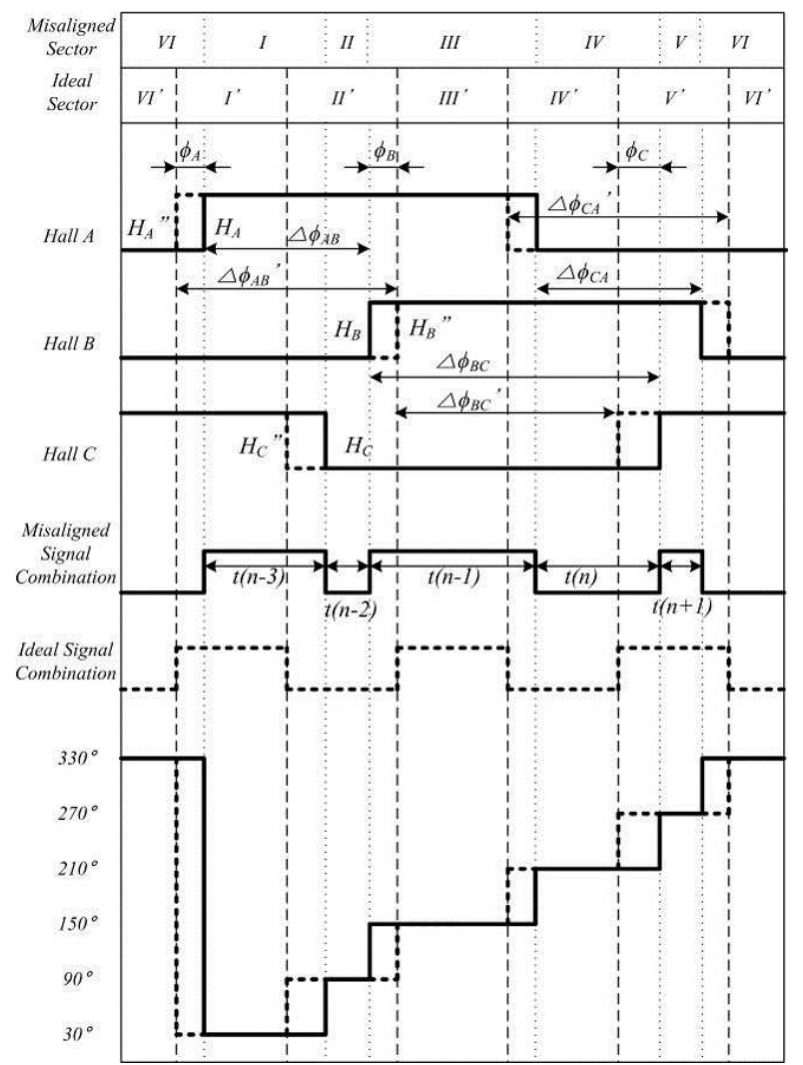

Fig. 2. Comparison of ideal and misaligned hall sensor signals.

$$
\phi_{n}=\phi_{r e}+\phi_{a e}
$$

where $n$ denotes sensors $\mathrm{A}, \mathrm{B}$, or $\mathrm{C}, \phi_{r e}$ is the relative position error, and $\phi_{a e}$ is the absolute position error. As the relative position errors of each hall-effect sensor are different, the relative position error can be derived as follows:

$$
\begin{aligned}
& \phi_{A}=\phi_{A}{ }^{\prime}+\phi_{a e} \\
& \phi_{B}=\phi_{B}{ }^{\prime}+\phi_{a e} \\
& \phi_{C}=\phi_{C}{ }^{\prime}+\phi_{a e}
\end{aligned}
$$

where $\phi_{A}{ }^{\prime}, \phi_{B}{ }^{\prime}$, and $\phi_{C}{ }^{\prime}$ are the relative errors of halleffect sensors $\mathrm{A}, \mathrm{B}$, and $\mathrm{C}$, respectively.

\subsection{Relative position estimation using least square method}

If the six output measurement sectors are classified according to the states of hall sensor signals, the rotor position can be easily estimated. Hall sensors that are positioned accurately will signal a phase difference of 120 degrees as follows:

$$
\Delta \phi^{\prime}=\Delta \phi_{A B}{ }^{\prime}=\Delta \phi_{B C}{ }^{\prime}=\Delta \phi_{C A}{ }^{\prime}=120^{\circ}
$$

where $\Delta \phi_{A B}{ }^{\prime}, \Delta \phi_{B C}{ }^{\prime}$ and $\Delta \phi_{C A}{ }^{\prime}$ are the respective differences produced by each ideal hall-effect sensor.

Under ideal operating conditions, $\Delta \phi_{A B}{ }^{\prime}, \Delta \phi_{B C}{ }^{\prime}$, and $\Delta \phi_{C A}{ }^{\prime}$ all have the same value, $\Delta \phi^{\prime}=120^{\circ}$. As shown in Fig. 2, the combination of the hall sensor output signals can be divided into six sectors, with each actual and ideal sector labeled as Sectors $m$ and $m$ ', respectively, where $m$ denotes Sectors I-VI. Using the intervals of Sectors I and II, Eq. (4) can be obtained; similarly, Eqs. (5) and (6) can be obtained from Sectors III-VI.

$$
\begin{aligned}
\Delta \phi_{A B}{ }^{\prime} & =\Delta \phi_{A B}+\phi_{A}-\phi_{B} \\
\Delta \phi_{B C}{ }^{\prime} & =\Delta \phi_{B C}+\phi_{B}-\phi_{C} \\
\Delta \phi_{C A}{ }^{\prime} & =\Delta \phi_{C A}+\phi_{C}-\phi_{A}
\end{aligned}
$$

Using equations (2) and (3), equations (4), (5) and (6) can be rewritten as follows:

$$
\begin{aligned}
& \Delta \phi^{\prime}=\Delta \phi_{A B}+\phi_{A}{ }^{\prime}-\phi_{B}{ }^{\prime} \\
& \Delta \phi^{\prime}=\Delta \phi_{B C}+\phi_{B}{ }^{\prime}-\phi_{C}{ }^{\prime} \\
& \Delta \phi^{\prime}=\Delta \phi_{C A}+\phi_{C}{ }^{\prime}-\phi_{A}{ }^{\prime}
\end{aligned}
$$

where $\Delta \phi_{A B}, \Delta \phi_{B C}$ and $\Delta \phi_{C A}$ are the actual invervals of each sector. Adding both sides of Eqs. (7), (8) and (9), Eq. (10) can be obtained as:

$$
3 \Delta \phi^{\prime}=\Delta \phi_{A B}+\Delta \phi_{B C}+\Delta \phi_{C A} .
$$


From the above equations, $\phi_{A}{ }^{\prime}, \phi_{B}{ }^{\prime}$, and $\phi_{C}{ }^{\prime}$ can be expressed as:

$$
\begin{gathered}
\phi_{A}{ }^{\prime}=\phi_{A}{ }^{\prime} \\
\phi_{B}{ }^{\prime}=\phi_{A}{ }^{\prime}+\Delta \phi_{A B}-\Delta \phi^{\prime} \\
\phi_{C}{ }^{\prime}=\phi_{A}{ }^{\prime}-\Delta \phi_{C A}+\Delta \phi^{\prime} .
\end{gathered}
$$

By using the least squares method $[10,11]$, the square of the each error and differentiation can be derived as follows:

$$
\begin{gathered}
E=\phi_{A}^{\prime 2}+\left(\phi_{A}{ }^{\prime}+\Delta \phi_{A B}-\Delta \phi^{\prime}\right)^{2}+\left(\phi_{A}{ }^{\prime}-\Delta \phi_{C A}+\Delta \phi^{\prime}\right)^{2} \\
\frac{\partial E}{\partial \phi_{A}{ }^{\prime}}=6 \phi_{A}{ }^{\prime}+2 \Delta \phi_{A B}-2 \Delta \phi_{C A}=0
\end{gathered}
$$

where $E$ is the square of each error. Then, the relative position becomes

$$
\begin{gathered}
\phi_{A}{ }^{\prime}=\frac{1}{3}\left(\Delta \phi_{A B}-\Delta \phi_{C A}\right) \\
\phi_{B}{ }^{\prime}=\frac{1}{3}\left(4 \Delta \phi_{A B}-\Delta \phi_{C A}-3 \Delta \phi^{\prime}\right) \\
\phi_{C}{ }^{\prime}=\frac{1}{3}\left(\Delta \phi_{A B}-4 \Delta \phi_{C A}+3 \Delta \phi^{\prime}\right) .
\end{gathered}
$$

In Eqs. (16), (17), and (18), $\Delta \phi_{A B}, \Delta \phi_{B C}$, and $\Delta \phi_{C A}$ can be obtained from actual hall sensor signals and $\Delta \phi^{\prime}$ is easily derivable by averaging motor speed. In the proposed relative error compensation method, balanced results are obtained from the misaligned hall sensors. Thus, if the absolute position error $\phi_{a e}$ is obtained, the total position error of the hall sensors can be estimated.

\subsection{Absolute position error compensation}

Estimating the position of the rotor is critical to the operation of a BLDC motor under sensorless control. Many methods for estimating rotor position have been proposed [13-17]; however, such methods usually require complicated computation, which can increase the relative cost of the system. The back-EMF sensing method is suitable for use on a wide range of motors because it requires no detailed knowledge of motor parameters. Back-EMF is relatively insensitive to motor manufacturing tolerance variation and is easily adaptable [18]. A typical BLDC motor is wound in a three-phase wye configuration in which one end of each phase is interconnected to a motor neutral point. During operation, only two of the three phases carry a connecting current (where the current flows in one phase and out the other) at any one time; this leaves one phase available to measure the back-EMF [16].

Fig. 3 shows the ZCP of the back-EMF and the signal of hall-effect sensor A. To measure the back-EMF, a virtual neutral point was built within a resistance network in which the voltage difference between the virtual neutral point and the open phase terminal could be sensed. By monitoring this voltage difference, the ZCP can be detected. Because there is a $30^{\circ}$ electrical offset between the ZCP of the back-EMF and the required commutation instant, the absolute position error $\phi_{a e}$ can be obtained.

Fig. 4 shows the detection of the ZCP at commutation Sector $\mathrm{k}$, where $\mathrm{k}$ is $1,2, \ldots, 6$. In this method, the motor terminal voltage is directly fed into the microcontroller through current-limit resistors and the back-EMF signal

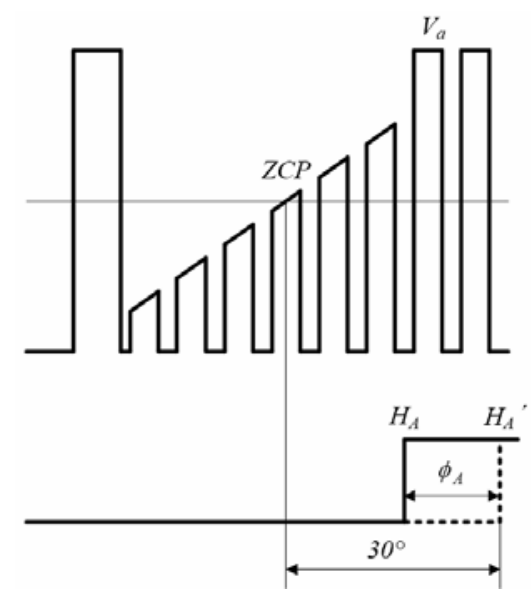

Fig. 3. ZCP and hall sensor signal comparison.

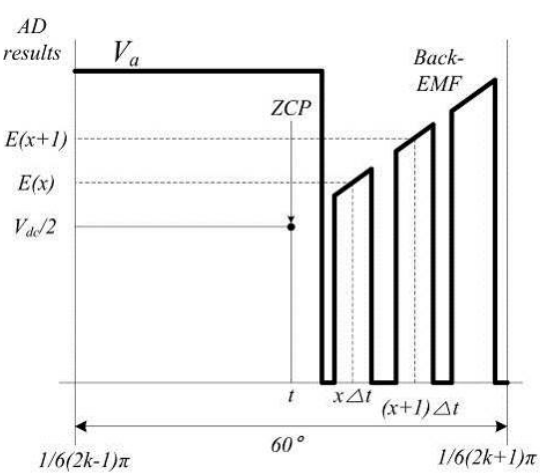

(a) Case I

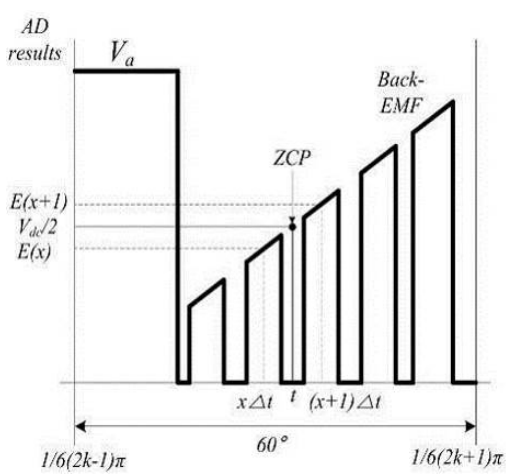

(b) Case II

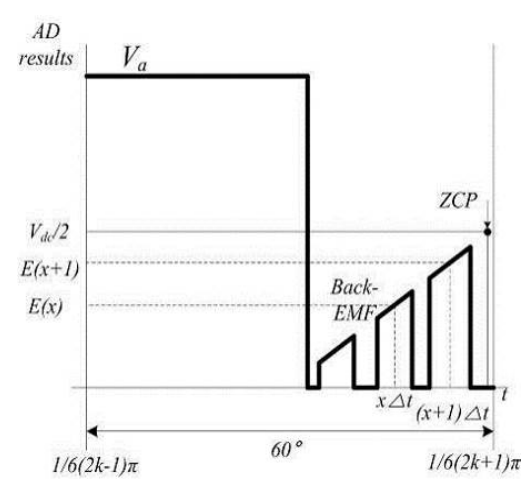

(c) Case III

Fig. 4. ZCP detection method. 
goes through an analog input channel, with the results of analog-to-digital (AD) conversion synchronized to a pulse width modulation (PWM) signal expressed as $E(x)$. The detection of the ZCP can be classified into three cases as given by the following equations:

$$
x \Delta t-t:(x+1) \Delta t-t=E(x)-\frac{V_{d c}}{2}: E(x+1)-\frac{V_{d c}}{2}
$$

where $t$ is the ZCP and $V_{d c}$ is the DC-link voltage of the drive. Using Eq. (15), Eq. (20) can be expressed as:

$$
t=\frac{x \Delta t E(x+1)-(x+1) \Delta t E(x)+\Delta t \frac{V_{d c}}{2}}{E(x+1)-E(x)}
$$

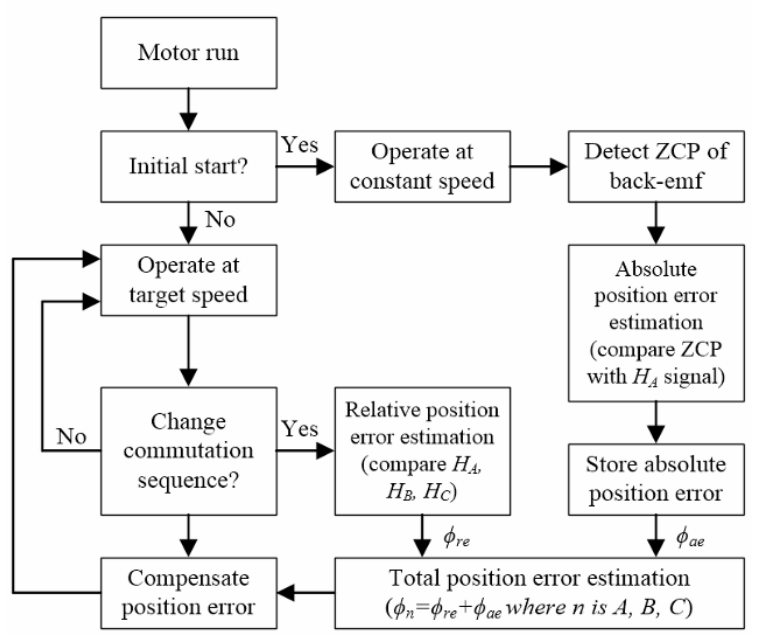

Fig. 5. Overall flowchart of position error estimation. where $E(x)$ and $E(x+1)$ can be obtained from the $\mathrm{AD}$ results. Thus, the absolute position error becomes

$$
t_{\phi_{a e}}=t_{30^{\circ}}-t
$$

where $t_{\phi_{a e}}$ is the time value of the absolute position error, and $t_{30^{\circ}}$ is the time value of $30^{\circ}$.

The total position error (2) can be obtained from the resultant position estimates of (16), (17), (18), and (21). The estimation of absolute position error on the drive, which is found by comparing the back-EMF to the hall sensor signal, is performed only once and then stored in flash memory. The overall control scheme for a BLDC motor drive with misaligned hall-effect sensors is shown in Fig. 6 and an overall flow chart of the position error estimation is shown in Fig. 5. In this process, the relative position error is estimated whenever the commutation sequence is changed. When the BLDC motor is first operated, the absolute position error is estimated at a specific constant speed and then stored in flash memory. The total position error is then calculated by adding the stored absolute error with the estimated relative position error. After initial operation, the final position is calculated using the stored absolute error. In this method, both the relative and absolute positions can be compensated.

\section{Experimental Results}

Fig. 8 shows an experimental setup for validating the proposed position compensation method, consisting of a prototype BLDC motor with misplaced hall sensors mounted on a printed circuit board placed outside of the motor case. Prior to describing the experimental results, it
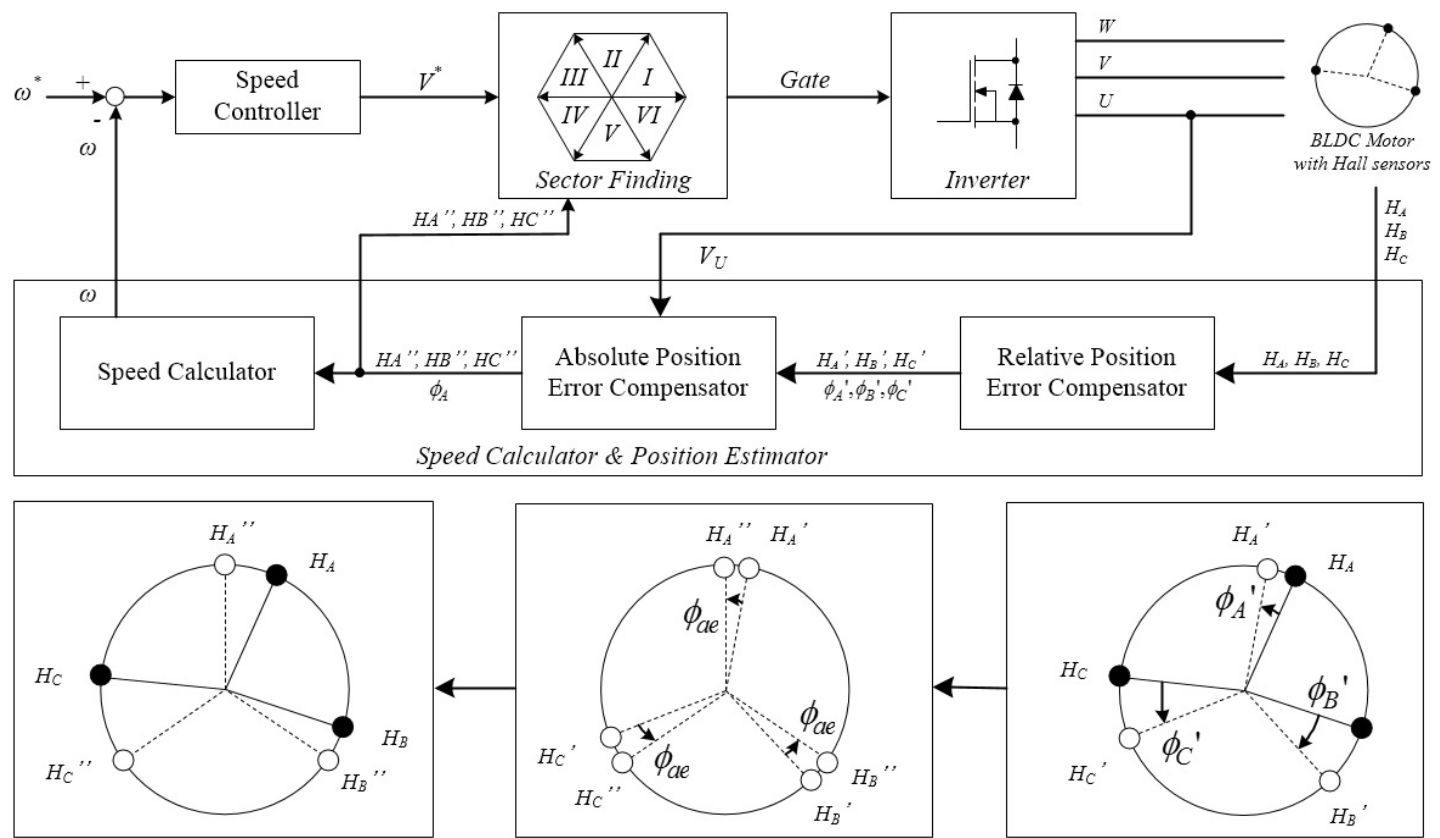

Fig. 6. ZCP and hall sensor signal comparison. 
Table 1. BLDC motor specifications.

\begin{tabular}{c|c|c}
\hline Items & Units & Specification \\
\hline Output power & $\mathrm{W}$ & 300 \\
\hline Number of poles & - & 20 \\
\hline Back-emf constant (peak to peak) & $\mathrm{V} / \mathrm{krpm}$ & 12 \\
\hline DC-link voltage & $\mathrm{V}$ & 48 \\
\hline Switching frequency & $\mathrm{kHz}$ & 20 \\
\hline $\begin{array}{c}\text { Circle diameter of hall-effect sensor } \\
\text { position }\end{array}$ & $\mathrm{mm}$ & 23 \\
\hline
\end{tabular}

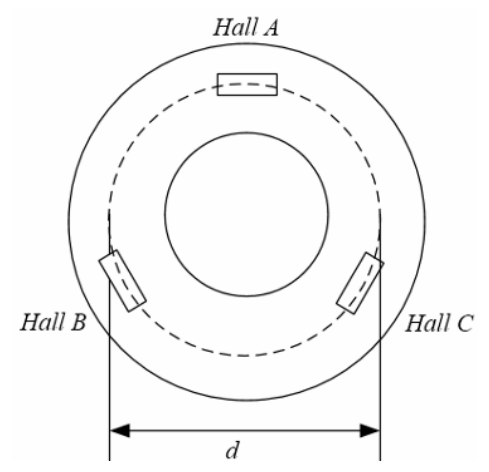

Fig. 7. Hall sensor board.
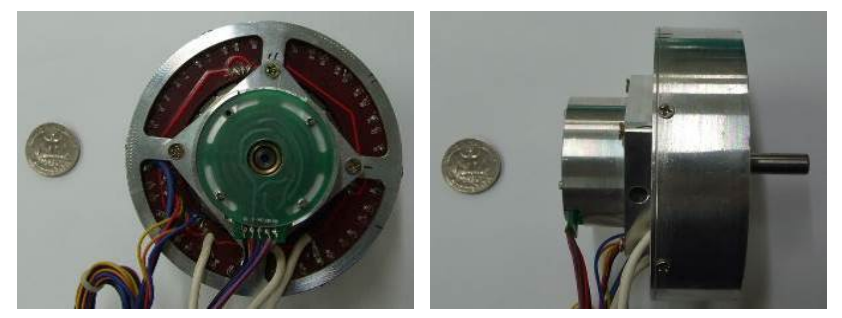

(a) BLDC motor and 25 cents

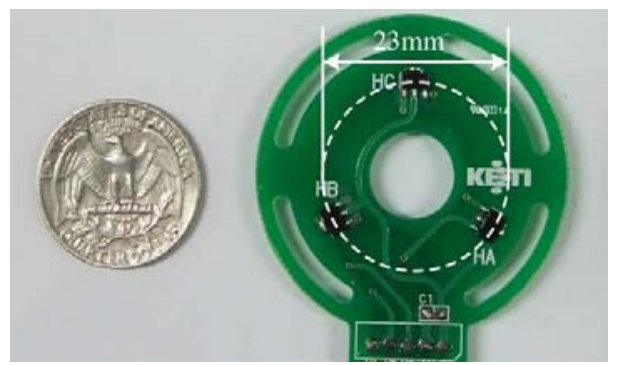

(b) Hall sensor board

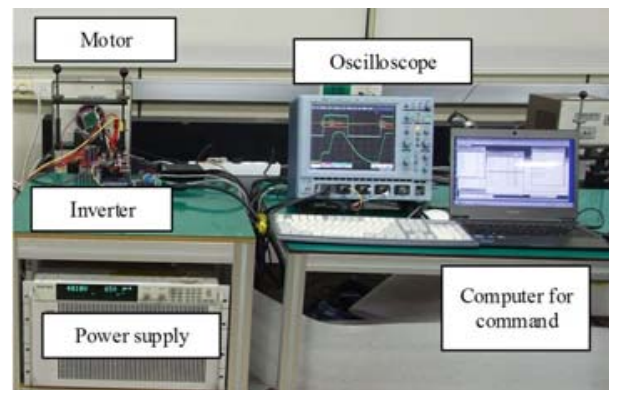

(c) Experimental setup

Fig. 8. The BLDC motor, hall sensor board, and experimental setup. is useful to provide some background on the BLDC motor. The test motor is a low-voltage, slim-type high-pole BLDC motor with 20 poles; its full specifications are shown in Table 1. Fig. 7 shows the hall sensor board. The angle of mechanical error $E_{h m}$ can be derived as follows:

$$
E_{h m}=\frac{360 x}{2 \pi \frac{d}{2}}
$$

where $x$ is the mechanical error of hall sensor position and $d$ is the circular diameter of the hall sensor position. Form the Eq. (22), the angle of electrical error $E_{h e}$ can be derived as follows:

$$
E_{h e}=\frac{P}{2} \frac{360 x}{2 \pi \frac{d}{2}}
$$

where $P$ is number of poles. Thus, the electrical error of hall sensor position is proportional to the number of poles and inversely proportional to the diameter. Because the circular diameter of a hall sensor position is approximately $23 \mathrm{~mm}$, if the error in positioning the hall sensor is $1 \mathrm{~mm}$, the mechanical degree of position error is $5^{\circ}$ and the electrical degree of position error is $50^{\circ}$. The PWM frequency of the inverter was set to $20 \mathrm{kHz}$ and the proposed position estimator was executed synchronously with the commutation period.

Fig. 9 shows the phase current waveform, position errors ( $\phi_{B}, \phi_{C}$ ), and motor speed at start-up operation. The differences between actual and ideal sensor placements were measured to be about $10.5^{\circ}, 16.9^{\circ}$, and $32.7^{\circ}$ for sensors A, B, and C, respectively. At first start-up, the ZCP detection algorithm was executed and the absolute position error was estimated and stored. The second start-up occurred without ZCP detection; instead, the inverter was operated using the stored absolute position errors. The position errors - about $16.9^{\circ}$ and $32.7^{\circ}$, respectively, as shown in Fig. 9 - had similar values to those from the first start-up.

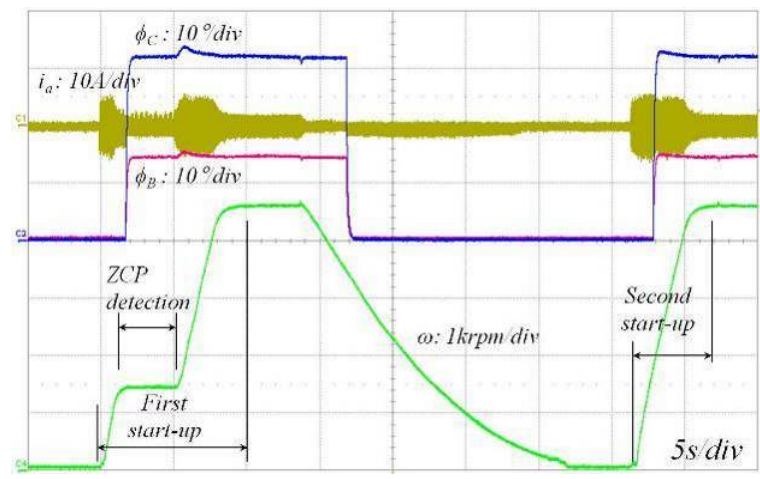

Fig. 9. Waveforms at start-up operation. 
Fig. 10 shows the current waveform, position errors $\left(\phi_{B}\right.$, $\left.\phi_{C}\right)$, and motor speed at start-up operation with a mechanical load. As in the previous case, the angular differences between actual and ideal sensor placements were measured to be about $10.5^{\circ}, 16.9^{\circ}$, and $32.7^{\circ}$ for sensors $\mathrm{A}, \mathrm{B}$, and $\mathrm{C}$, respectively. The load was increased to the $100 \%$ level of $0.8 \mathrm{Nm}$. The position errors, as can be seen in Fig. 9, remained when the load was changed, demonstrating that the proposed method has good transient performance in achieving position compensation.

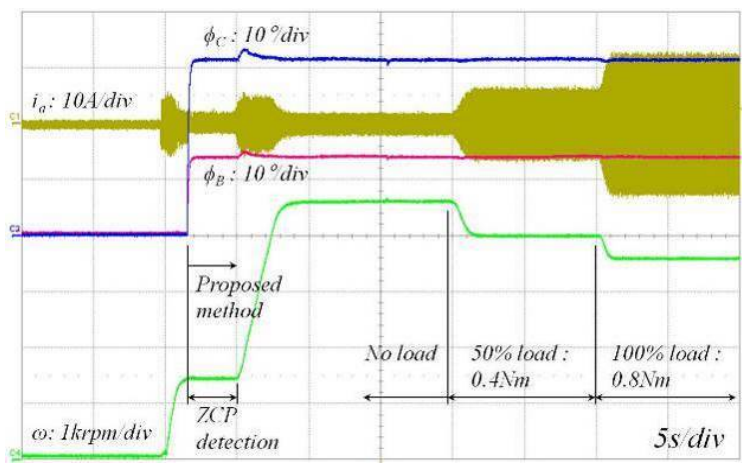

Fig. 10. Waveforms with a mechanical load.

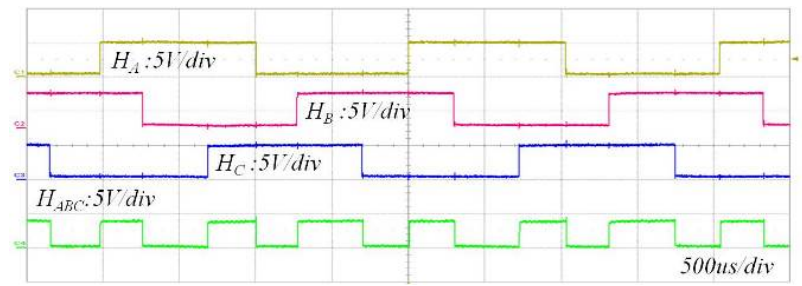

(a) Hall sensor signals and combination signal.

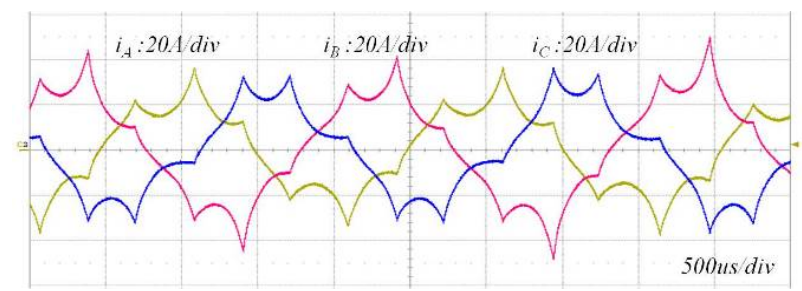

(c) Phase current waveforms without proposed method.
Several experiments were performed to validate the proposed position estimator. Each experiment tested a misaligned case, as shown in Table 2, where the angles shown represent the electrical degree of position error, the + mark refers to leaded position, and the - mark denotes the lagging position. In case 1, the BLDC motor was operated counterclockwise (CCW). In this case, the maximum relative position error was as small as $13.4^{\circ}$ and the positions of all hall sensors were lagged. In case 2, the motor was operated clockwise (CW) with a maximum relative position error at a large value of $52.1^{\circ}$ and hall sensor B was in the leading position while sensors A and C were lagging.

\subsection{Case 1 (Relative position error : $\mathbf{1 0}^{\circ}$ )}

The characteristics of the proposed approach can be assessed through a comparative study of the results of each

Table 2. Two test cases of the proposed method.

\begin{tabular}{c|c|c|c|c|c}
\hline & Dir. & $\begin{array}{c}\text { Max. relative } \\
\text { error }\end{array}$ & $\begin{array}{c}\text { Hall A } \\
\text { error }\end{array}$ & $\begin{array}{c}\text { Hall } \\
\text { B error }\end{array}$ & $\begin{array}{c}\text { Hall } \\
\text { C error }\end{array}$ \\
\hline Case 1 & CCW & $13.4^{\circ}$ & $-21.1^{\circ}$ & $-17.5^{\circ}$ & $-7.7^{\circ}$ \\
\hline Case 2 & CW & $52.1^{\circ}$ & $-3.7^{\circ}$ & $+26.2^{\circ}$ & $-25.9^{\circ}$ \\
\hline
\end{tabular}

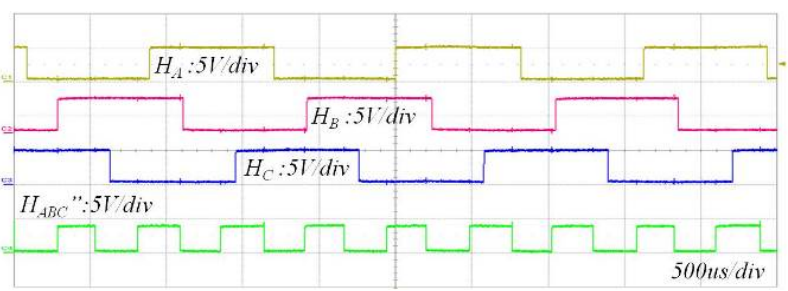

(b) Hall sensor signals and estimated combination signal.

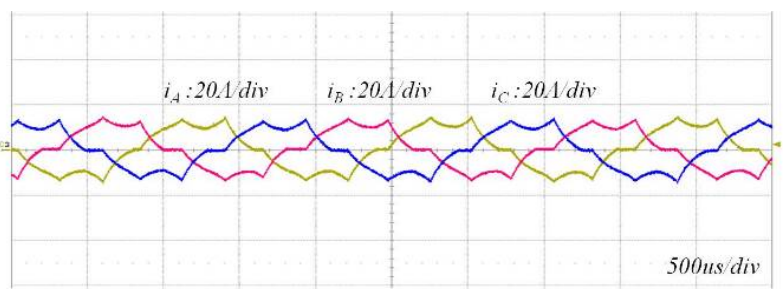

(d) Phase current waveforms with proposed method.
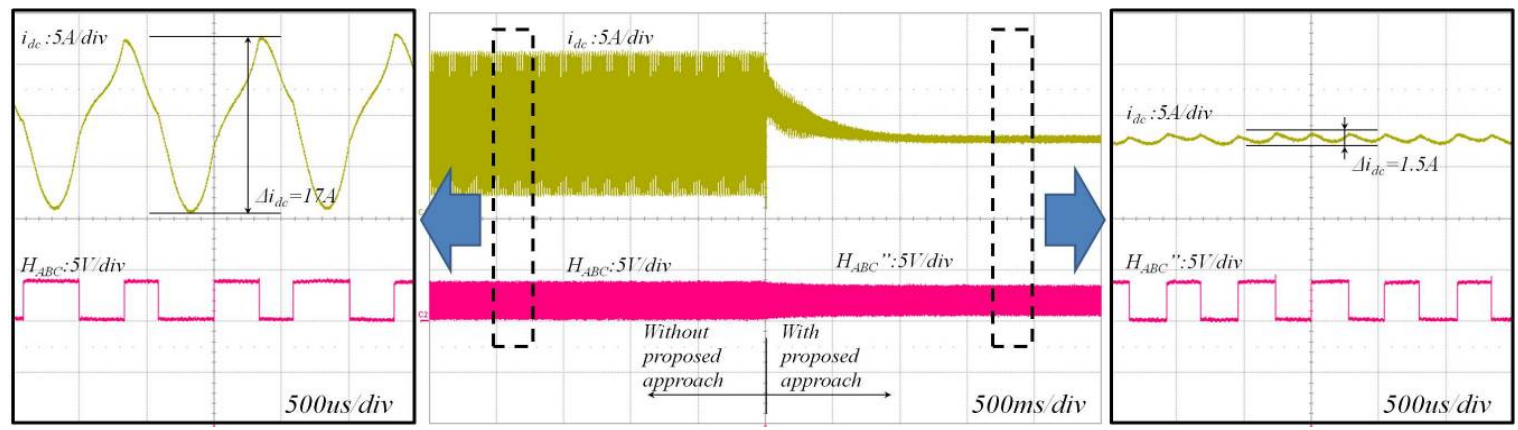

(e) Input current waveforms, combination signal and estimated combination signal of hall sensors.

Fig. 11. Waveforms at $100 \%$ load $(0.8 \mathrm{Nm})$ in case 1. 
case. Table 3 shows the hall sensor error for case 1 , where the angular differences in actual sensor placements are measured to be about $-21.1^{\circ},-17.5^{\circ}$, and $-7.7^{\circ}$ for Sensors A, $\mathrm{B}$, and $\mathrm{C}$, respectively. The signals of all hall sensors are in a lagging position in which the maximum relative electrical angle difference is about $10^{\circ}$ and the mechanical angular difference is about $1^{\circ}$, as the test motor has 20 poles. The hall sensor board has a circular diameter of about $23 \mathrm{~mm}$ and a circumference of hall sensor position of about $72 \mathrm{~mm}$. In case 1 , the maximum error in positioning of the hall sensors is about $0.2 \mathrm{~mm}$, meaning that approximate errors of $0.2 \mathrm{~mm}$ are likely to occur during the process of production.

Table 3. Hall sensor error for case 1.

\begin{tabular}{c|c|c|c|c|c}
\hline & Dir. & $\begin{array}{c}\text { Max. relative } \\
\text { error }\end{array}$ & $\begin{array}{c}\text { Hall A } \\
\text { error }\end{array}$ & $\begin{array}{c}\text { Hall B } \\
\text { error }\end{array}$ & $\begin{array}{c}\text { Hall C } \\
\text { error }\end{array}$ \\
\hline Case 1 & CCW & $13.4^{\circ}$ & $-21.1^{\circ}$ & $-17.5^{\circ}$ & $-7.7^{\circ}$ \\
\hline
\end{tabular}

Fig. 11 shows the individual hall sensor output signals, the actual combined signal produced by the hall sensors $\left(H_{A B C}\right)$, the estimated combined signal of the sensors $\left(H_{A B C}\right.$ "), the phase current, and the input current at $0.8 \mathrm{Nm}$ load, for two cases, i.e., with and without the application of

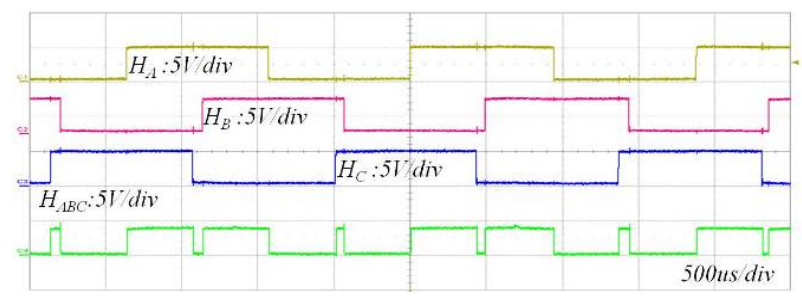

(a) Hall sensor signals and combination signal.

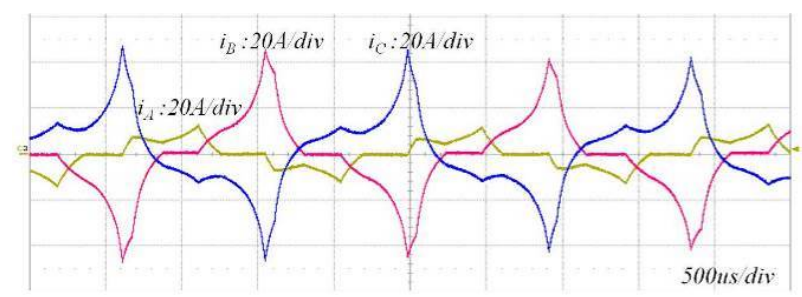

(c) Phase current waveforms without proposed method. the proposed approach. Fig. 11 (a) shows that the combination of hall sensor signals does not have a uniform waveform and Fig. 11 (c) shows that the phase current waveforms without the application of the proposed approach are seriously distorted at the end of every combination of the hall sensor signals. This is due to the misalignment of the hall sensors. Fig. 11 (b) shows the estimated combination signal of the hall sensors that has uniform $60^{\circ}$ sectors. Fig. 11 (d) shows the phase current waveforms with the application of the proposed method. Every phase current has uniform waveforms. In Fig. 11 (e), it is seen that the input current without the application of the proposed approach is seriously distorted at the end of every combination of the hall sensor signals. On the contrary, the results of the application of the proposed method in Fig. 11 (e) show that ripples of the current waveforms are significantly reduced and regularly generated compared to those without the application of proposed method.

\subsection{Case 2 (Relative position error : $50^{\circ}$ )}

Table 4 shows the hall sensor errors in case 2. The respective differences between actual and ideal angles have

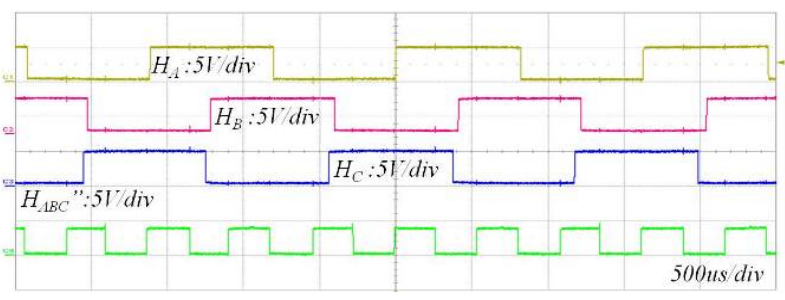

(b) Hall sensor signals and estimated combination signal.

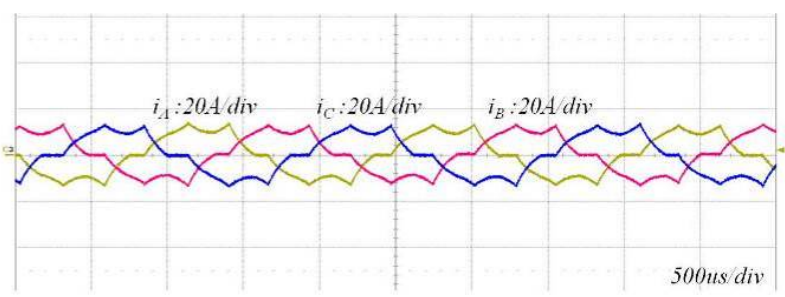

(d) Phase current waveforms with proposed method.
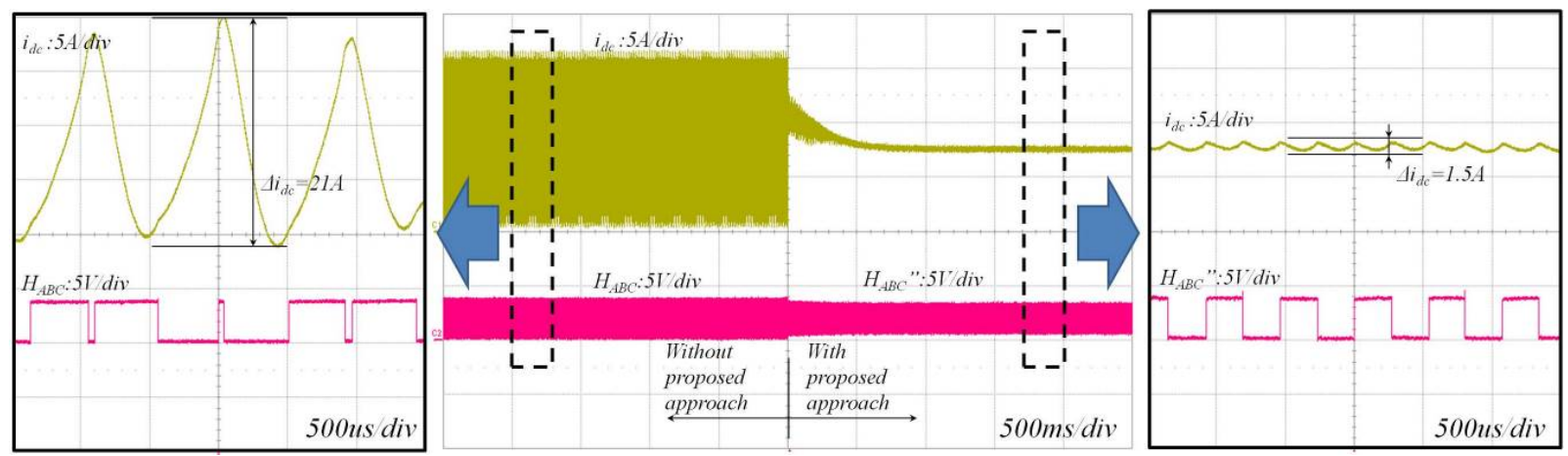

(e) Input current waveforms, combination signal and estimated combination signal of hall sensors.

Fig. 12. Waveforms at $100 \%$ load $(0.8 \mathrm{Nm})$ in case 2. 
Table 4. Hall sensor error for case 2.

\begin{tabular}{c|c|c|c|c|c}
\hline & Dir. & $\begin{array}{c}\text { Max. relative } \\
\text { error }\end{array}$ & $\begin{array}{c}\text { Hall A } \\
\text { error }\end{array}$ & $\begin{array}{c}\text { Hall B } \\
\text { error }\end{array}$ & $\begin{array}{c}\text { Hall C } \\
\text { error }\end{array}$ \\
\hline Case 2 & CW & $52.1^{\circ}$ & $-3.7^{\circ}$ & $+26.2^{\circ}$ & $-25.9^{\circ}$ \\
\hline
\end{tabular}

been measured to be about $-3.7^{\circ},+26.2^{\circ}$, and $-25.9^{\circ}$. The signal of hall sensor $\mathrm{B}$ is leaded while those of $\mathrm{A}$ and $\mathrm{C}$ rests are lagged. The maximum relative electrical angle difference is about $50^{\circ}$ and the mechanical angle difference is about $5^{\circ}$. As in case 1 , the hall sensor board has a circle diameter of about $23 \mathrm{~mm}$ and a circumference of hall sensor positions of about $72 \mathrm{~mm}$. The maximum error in positioning the hall sensors is about $1.0 \mathrm{~mm}$, which, though large compared with the error in case 1 , can still occur with significant frequency during the production process.

Fig. 12 shows, both with and without the proposed approach, the hall sensor signals, the actual combined sensor signal, the estimated combined signal of the sensors, the phase current, and the input current at $0.8 \mathrm{Nm}$ load. In Fig. 12 (a), it is seen that the combined hall sensor signal does not have uniform $60^{\circ}$ sectors; in particular, the signals of hall sensors B and C are nearly inverted. In Fig. 12 (c), it is seen that the phase current waveforms produced without using the proposed approach are seriously distorted at the ends of the sensor B and C signals. This is caused by effects originating in the misalignment of the hall sensors. As shown in Table 4, although hall sensor A is nearly correct, sensors B and C have significant errors. Fig. 12 (b) shows the estimated combined signal of the hall sensors; as in case 1 , the final result of the estimated hall signal has uniform $60^{\circ}$ sectors. Fig. 12 (d) shows the phase current waveforms produced using the proposed method. Every phase current has a uniform waveform, results that are the same as in case 1. In Fig. 12 (e), it is seen that the input current produced without using the proposed approach is seriously distorted. By contrast, the results produced by the proposed method [Fig. 12 (e)] demonstrate a more regularly generated input ripple with a significantly reduced magnitude of $1.5 \mathrm{~A}$.

\section{Conclusion}

In this paper, a position error compensation method to derive the rotor position in BLDC drives with misaligned hall sensors was proposed. This approach estimated relative positions using hall sensor signals and absolute positions using the back-EMF signal of the phase voltage. The proposed method provides accurate position information even if the sensor error is relatively large. By using this method, the current ripple of a BLDC motor can be reduced significantly under the two test condition cases with errors of $10^{\circ}$ and $50^{\circ}$, respectively. The performance of the proposed position estimator was experimentally verified.

\section{Acknowledgements}

This work was supported by the National Research Foundation of Korea (NRF) grant funded by the Korea government (MSIP) (No. 2013R1A2A1A01015171)

\section{Reference}

[1] T. J. E. Miller, and Hendershot, Design of brushless permanent magnet motors, Magna Physics publicshing and Clarendon Press, Oxford, 1994.

[2] Richard Valentine, Motor control electronics handbook, McGrow-Hill Handbook, 1998.

[3] P. C. Krause, O. Wasynczuk, and S. D. Sudhoff, Analysis of electric machinery and drive systems, IEEE Press, Piscataway, NJ, 2002.

[4] P. Alaeinovin, S. Chiniforoosh, and J. Jatskevich, "Evaluating misalignment of hall sensors in brushless DC motors," proc. of IEEE Electric Power Conference, pp. 1-6, 2008.

[5] N. Samoylenko, Q. Han, and J. Jatskevich, "Dynamic performance of brushless DC motors with unbalanced hall sensors," IEEE Trans. Energy Conversion, vol. 23, Iss. 3, pp. 752-763, 2008.

[6] P. Alaeinovin, and J. Jatskevich, "Hall-sensor signals filtering for improved operation of brushless DC motors," proc. of IEEE ISIE, pp. 613-618, 2011.

[7] P. Alaeinovin, and J. Jatskevich, "Filtering of hallsensor signals for improved operation of brushless DC motors," IEEE Trans. Energy Conversion, vol. PP, Iss. 99, pp. 1-3, 2012.

[8] S.Y. Kim, C.Choi, K.Lee, and W. Lee, “An improved rotor position estimation with vector tracking observer in PMSM drives with low resolution halleffect sensors," IEEE Trans. Industrial Electronics, vol.58, Iss. 9, pp. 4078-4086, 2011.

[9] F. Giulii Capponi, G. De Donato, L. Del Ferraro, O. Honorati, M. C. Harke, and R. D. Lorenz, "AC brushless drive with low-resolution hall-effect sensors for surface-mounted PM machines," IEEE Trans. Ind. Appl., vol. 42, no. 2, pp. 526-535, 2006.

[10] Dong-Gyu Lee, Sang-Hee Kang, and Soon-Ryul Nam, "Phaser estimation algorithm based on the least square technique during CT saturation”, Journal of Electrical Engineering \& Technology, vol. 6, Iss. 4, pp. 459-465, 2011.

[11] Hong Zheng, Zheng-feng Liang, Meng-shu Li, and Kai Li, "Optimization of parameters for LCL filter of least square method based three-phase PWM converter," Journal of Electrical Engineering \& Technology, vol. 10, Iss. 4, pp. 1626-1634, 2015.

[12] J. H. Choi, J. S. Park, B. G. Gu, J. H. Kim, and C. Y. Won, "Position estimation and control of BLDC motor for VVA module with unbalanced hall sensors," proc. of IEEE PECON, pp. 390-395, 2012. 
[13] S. Ogasawara and H. Akai, "An approach to position sensorless drive for brushless DC motors," IEEE Trans. Ind. Appl., vol. 27, Iss. 5, pp. 928-923, 1991.

[14] N. Ertugrul and P. Acarmley, "A new algorithm for sensorless operation of permanent magnet motors," IEEE Trans. Ind. Appl., vol. 30, Iss. 1, pp. 126-133, 1994.

[15] P. Pillay and R. Krishnan, "Modeling, simulation, and analysis of permanent-magnet motor drives, Part II: The brushless DC motor drive,” IEEE Trans. Ind. Appl., vol. 25, Iss. 2, pp. 274-279, 1989.

[16] Jianwen Shao, D. Nolan, M. Teissier, and D. Swanson, "A novel microcontroller-based sensorless brushless DC (BLDC) motor drive for automotive fuel pumps,” IEEE Trans. Ind. Appl., vol. 39, Iss. 6, pp. 1734-1740, 2003.

[17] Hui T. S., Basu K. P., and Subbiah V., "Permanent magnet brushless motor control techniques,” proc. of IEEE PECON, pp. 133-138, 2003.

[18] K. S. Rama Rao, Nagadeven, and S. Taib, "Sensorless control of a BLDC motor with back EMF detection method using DSPIC," proc. of IEEE PECON, pp. 243-248, 2008.

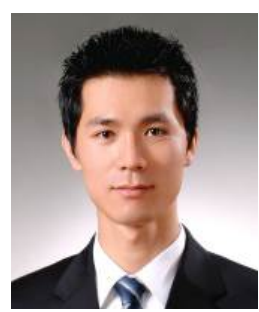

Joon Sung Park He was born in Seoul, Korea, in 1978. He received B. S. degree in Electrical and Electronic Engineering from Hongik University, Seoul, Korea, in 2005, and his M.S. degree in Electrical and Electronic Engineering from Pohang University of Science and Technology (POSTECH), Pohang, Korea, in 2007. Since 2015, he has been pursuing the Ph.D. degree at the Department of Electrical Engineering, Hanyang University, Seoul, Korea. He is also currently a Senior Research Engineer at KETI (Korea Electronics Technology Institute), Bucheon, Korea. His current research interests include AC motor control, PWM converter/inverter system, and wave energy converter.

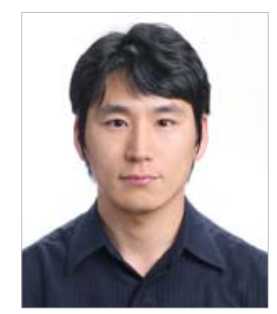

Jun-Hyuk Choi He was born in Daegu, Korea, in 1974. He received M. S. and Ph.D. from the School of Electrical and Electronic Engineering, Sungkyunkwan University, Suwon, Korea, in 2003 and 2013, respectively. He is currently Managerial Research Engineer at KETI (Korea Electronics Technology Institute), Bucheon, Korea. His current research interests include power electronics and motor control systems.

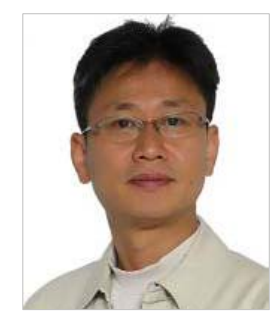

Ju Lee He received his M.S. degree from Hanyang University, Seoul, South Korea, in 1988, and his Ph.D. from Kyusyu University, Japan in 1997, both in Electrical Engineering, $\mathrm{He}$ joined Hanyang University in September, 1997 and is currently a Professor of the Division of Electrical and Biomedical Engineering. His main research interests include electric machinery and its drives, electromagnetic field analysis, new transformation systems such as hybrid electric vehicles (HEV), and high-speed electric trains and standardization. He is a member of the IEEE Industry Applications Society, Magnetics Society, and Power Electronics Society. 